\title{
AN INTEGRAL CRITERION FOR AUTOMORPHIC AND ROTATION AUTOMORPHIC FUNCTIONS
}

\author{
Rauno Aulaskari, Walter K. Hayman, and Peter Lappan
}

\section{Introduction}

Let $D=\{z:|z|<1\}$ denote the unit disk in the complex plane and let $\Gamma$ denote a Fuchsian group acting on $D$. Let $F$ denote the fundamental region of $\Gamma$. We may think of $F$ as the "Ford fundamental region" $F_{0}=\{z \in D:|z| \leq|\gamma(z)|$ for each $\gamma \in \Gamma$, but any choice of a fundamental region $F$ for which the area of the boundary of $F$ is zero will serve our purposes for most of this paper. We say that the function $f$ meromorphic in $D$ is an automorphic function (with respect to the Fuchsian group $\Gamma$ ) if $f(\gamma(z))=f(z)$ for each $\gamma \in \Gamma$ and each $z \in D$. We say that a meromorphic function $f$ is a rotation automorphic function (with respect to $\Gamma$ ) if for each $\gamma \in \Gamma$ there exists a rotation $S_{\gamma}$ of the Riemann sphere (with radius $1 / 2$ ) such that $f(\gamma(z))=S_{\gamma}(f(z))$ for each $z \in D$. Also, we say that an analytic function $f$ is an additive automorphic function (with respect to $\Gamma$ ) if for each $\gamma \in \Gamma$ there exists a complex number $A_{\gamma}$ such that $f(\gamma(z))=f(z)+A_{\gamma}$ for each $z \in D$.

Let $f^{\#}(z)=\left|f^{\prime}(z)\right| /\left(1+|f(z)|^{2}\right)$ denote the spherical derivative of $f$. We say that a meromorphic function $f$ is a normal function in $D$ if

$$
\sup \left\{\left(1-|z|^{2}\right) f^{\#}(z): z \in D\right\}<\infty
$$

and we denote the class of normal functions by $\mathcal{N}$ (see [14]). We say that an analytic function $f$ is a Bloch function if

$$
\sup \left\{\left(1-|z|^{2}\right)\left|f^{\prime}(z)\right|: z \in D\right\}<\infty
$$

and we denote the class of Bloch functions by $\mathcal{B}$ (see [18]). It is clear that $\mathcal{B} \subset \mathcal{N}$.

For a Fuchsian group $\Gamma$, we say that an analytic function $g$ is an automorphic form of weight 1 if $g(\gamma(z)) \gamma^{\prime}(z)=g(z)$ for each $\gamma \in \Gamma$ and each $z \in D$. It is easy to see that if $f$ is an additive automorphic function then $f^{\prime}$ is an automorphic

The first author was supported in part by grant 1021005 from the Academy of Finland. 
form of weight 1 . For $1 \leq p<\infty$, we denote by $A^{p}(\Gamma)$ the collection of all automorphic forms of weight 1 which satisfy the condition

$$
\iint_{F}\left(1-|z|^{2}\right)^{p-2}|g(z)|^{p} d x d y<\infty
$$

and, for $p=\infty$, we denote by $A^{\infty}(\Gamma)$ the collection of all automorphic forms of weight 1 which satisfy the condition

$$
\sup \left\{\left(1-|z|^{2}\right)|g(z)|: z \in F\right\}<\infty .
$$

The Banach spaces $A^{p}(\Gamma), 1 \leq p \leq \infty$, are known as the Bers spaces. It is obvious that if $f$ is an additive automorphic function then $f^{\prime} \in A^{\infty}(\Gamma)$ if and only if $f \in \mathcal{B}$.

The Bers spaces $A^{p}(\Gamma)$ have been very useful in the modern theory of Riemann surfaces. In particular, there was much interest in the so-called "Bers conjecture": is $A^{p}(\Gamma) \subset A^{\infty}(\Gamma)$ ? This conjecture was disproved by Pommerenke [18, Theorem 2] in 1976 when he constructed an additive automorphic function $f$ such that $f^{\prime} \in A^{2}(\Gamma)-A^{\infty}(\Gamma)$. However, several authors, among them Drasin and Earle [6], Lehner [11], Metzger and Rao [15], [16], and Knopp [10], all proved some version of the following result: if $f$ is an additive automorphic function with respect to a finitely generated Fuchsian group $\Gamma$ and if $g=f^{\prime}$ satisfies (1), then $f \in \mathcal{B}$. In the case of infinitely generated Fuchsian groups, Lehner [12] and [13] proved a similar result under the assumption that the fundamental region $F$ is not too "thin" in the sense of the hyperbolic metric (except possibly near parabolic vertices). Finally, Niebur and Sheingorn [17] gave a complete description for when the Bers conjecture is valid. Their description says, in effect, that the Bers conjecture has an affirmative answer if and only if the fundamental region is not too "thin" in Lehner's sense.

We observe that the Bers conjecture was really a question about additive automorphic functions. In this paper we address a similar general question about rotation automorphic functions. Here, the class $\mathcal{N}$ of normal functions plays the role of $\mathcal{B}$, which is equivalent to $A^{\infty}(\Gamma)$ in the sense described above. We look at the following question: if $f$ is a rotation automorphic function which satisfies the condition

$$
\iint_{F}\left(1-|z|^{2}\right)^{p-2}\left(f^{\#}(z)\right)^{p} d x d y<\infty
$$

for $p \geq 2$, under what conditions is $f \in \mathcal{N}$ ? In [4, Corollary 3, p. 88], the first and third authors gave an example of a rotation automorphic function $f$ which is not a normal function but which satisfies (2) with $p=2$. Pommerenke [19, Corollary, pp. 194-195] has proved that an automorphic function $f$ which satisfies 
(2) for $p=2$ is a normal function. In Section 2 we prove that Pommerenke's result is valid for all $p \geq 2$, that is, if an automorphic function satisfies (2) for some $p \geq 2$, then it is a normal function. In fact, our result gives some additional information about the bound on the expression $\left(1-|z|^{2}\right) f^{\#}(z)$ in the case $p>2$. In Section 3 we prove two results giving other positive answers to our question. We show that if the fundamental region satisfies some mild "thinness" conditions due to Lehner, and $f$ is a rotation automorphic function, then (2) implies that $f$ is a normal function. And also, if $\Gamma$ is a finitely generated group and $f$ is a rotation automorphic function which satisfies (2), then $f$ is a normal function and, in addition, we have $\left(1-|z|^{2}\right) f^{\#}(z) \rightarrow 0$ as $|z| \rightarrow 1$ from within $F$.

In Sections 4 and 5 we give some results suggested by a result of Metzger and Rao [15, Theorem 3]: If $f$ is an additive automorphic function with respect to a finitely generated Fuchsian group $\Gamma$ of the second kind, and if

$$
\iint_{F} \frac{\left|f^{\prime}(z)\right|}{1-|z|^{2}} d x d y<\infty
$$

then $f$ is a constant function. In Section 4, we prove that if $f$ is a meromorphic function satisfying

$$
\iint_{D} \frac{\left(f^{\#}(z)\right)^{p}}{1-|z|^{2}} d x d y<\infty
$$

where $p \geq 1$, then either $f$ is a constant or else $\lim \sup _{z \rightarrow \zeta}\left(1-|z|^{2}\right) f^{\#}(z)=\infty$ for each point $\zeta \in \partial D$. If $p \geq 2$ here, then $f$ must be a constant. We note that if $f$ is rotation automorphic and satisfies condition (3) for $p=1$, then we have

$$
\iint_{D} \frac{f^{\#}(z)}{1-|z|^{2}} d x d y=\sum_{\gamma \in \Gamma} \iint_{\gamma(F)} \frac{f^{\#}(z)}{1-|z|^{2}} d x d y<\infty
$$

and since each of the integrals in the sum on the right have the same value, we have that either $\Gamma$ contains only a finite number of elements or else $f$ must be constant. Thus, our result is related to but somewhat different from the Metzger and Rao result.

In Section 5, we show that examples of non-constant meromorphic functions satisfying (3) exist for each $p$ satisfying $0<p<2$. In fact, what is actually shown is a somewhat more general result, where the integrand in (3) is of a more general form. The example given is an automorphic function for the finitely generated rotation group $\left(\Gamma=\left\{\right.\right.$ identity, $\left.\gamma_{1}\right\}$, where $\left.\gamma_{1}(z)=-z\right)$, which shows that the analogue to the Metzger and Rao result is not valid for automorphic functions (or rotation automorphic functions) for which the group $\Gamma$ has a finite number of elements.

Finally, in Section 6 we clarify by an example a result from Section 2 about the class of automorphic functions which satisfy (2). 


\section{The case of automorphic functions}

In this section we prove the following result.

Theorem 1. Let $f$ be a meromorphic function in $D$ which is automorphic with respect to a Fuchsian group $\Gamma$, and define

$$
I=\iint_{F}\left(1-|z|^{2}\right)^{p-2}\left(f^{\#}(z)\right)^{p} d x d y
$$

If $p \geq 2$ and $I<\infty$, then $f$ is a normal function. In fact, if $p>2$, then we have that

$$
\sup \left\{\left(1-|z|^{2}\right) f^{\#}(z): z \in D\right\} \leq 3 K=3 \max \left\{I^{1 / p}, I^{1 /(p-2)}\right\} .
$$

Since Pommerenke [19] has proved the Theorem for the case $p=2$, we need to deal only with the case $p>2$. In the proof, we will make use of the following result of J. Dufresnoy [7, Lemma II, p. 216].

Lemma 1. If the function $f$ is meromorphic in the disk $D_{r_{0}}=\left\{z:|z|<r_{0}\right\}$, and if the area on the Riemann sphere of the set $f\left(D_{r_{0}}\right)$ is less than $\sigma_{0} \pi$, where $0<\sigma_{0}<1$, then

$$
\left(f^{\#}(0)\right)^{2} \leq \frac{1}{r_{0}^{2}} \frac{\sigma_{0}}{1-\sigma_{0}} .
$$

We remark that the Riemann sphere we are using has radius $1 / 2$ and thus has total area $\pi$.

Proof of Theorem 1. Let $E$ be the set of all points $z \in D$ for which

$$
\left(1-|z|^{2}\right) f^{\#}(z) \geq K>0
$$

(we may suppose that $f$ is not a constant function) and we define $E_{0}=E \cap F$. Since $f$ is automorphic with respect to the Fuchsian group $\Gamma$, we have that $f(E)=$ $f\left(E_{0}\right)$. Let $S^{\prime}$ denote the spherical area of $f\left(E_{0}\right)$. If $d m(z)=\left(1-|z|^{2}\right)^{-2} d x d y$ denotes the element of hyperbolic area, we have

$$
\begin{aligned}
S^{\prime} & \leq \iint_{E_{0}}\left\{\left(1-|z|^{2}\right) f^{\#}(z)\right\}^{2} d m(z) \\
& \leq K^{2-p} \iint_{E_{0}}\left\{\left(1-|z|^{2}\right) f^{\#}(z)\right\}^{p} d m(z) \leq K^{2-p} I \leq 1
\end{aligned}
$$

because of (4) and the definition of $K$. We now define $\varrho$ by the equation

$$
I K^{2-p}=\frac{\pi K^{2} \varrho^{2}}{1-\varrho^{2}}
$$


and define $D_{\varrho}=\{z:|z|<\varrho\}, A=D_{\varrho} \cap E$, and $B=D_{\varrho}-A$. The spherical area of $f(B)$ is at most

$$
\begin{aligned}
\iint_{B}\left(f^{\#}(z)\right)^{2} d x d y & <\iint_{B} \frac{K^{2}}{\left(1-|z|^{2}\right)^{2}} d x d y \\
& \leq K^{2} \int_{0}^{\varrho} \int_{0}^{2 \pi} \frac{r d r d \theta}{\left(1-r^{2}\right)^{2}}=\frac{\pi K^{2} \varrho^{2}}{1-\varrho^{2}}=I K^{2-p} \leq 1
\end{aligned}
$$

Thus, the spherical area $S_{0}$ of $f\left(D_{\varrho}\right)$ is at most $2 I K^{2-p} \leq 2$. From Lemma 1 , we have that

$$
\left(f^{\#}(0)\right)^{2} \leq \frac{1}{\varrho^{2}} \frac{S_{0}}{\pi-S_{0}} \leq \frac{2 I K^{2-p}}{\varrho^{2}}
$$

If $I \geq 1$, then we have $K=I^{1 /(p-2)}$, and (6) yields that

$$
\frac{1}{\varrho^{2}}-1=\frac{\pi K^{p}}{I}=\pi I^{p /(p-2)-1}=\pi I^{2 /(p-2)} \geq \pi
$$

Thus we have

$$
\frac{2 I K^{2-p}}{\varrho^{2}}=2\left(1+\pi I^{2 /(p-2)}\right) \leq 2(1+\pi) I^{2 /(p-2)}<9 I^{2 /(p-2)} .
$$

It follows that

$$
f^{\#}(0) \leq \sqrt{9 I^{2 /(p-2)}}=3 I^{1 /(p-2)}=3 K
$$

from (7).

If $I<1$, then $K=I^{1 / p}$ and

$$
\frac{1}{\varrho^{2}}-1=\frac{\pi K^{p}}{I}=\pi
$$

so

$$
\frac{2 I K^{2-p}}{\varrho^{2}}=2(\pi+1) I^{(1+(2-p) / p)}<9 I^{2 / p}
$$

and again, $f^{\#}(0) \leq 3 I^{1 / p}=3 K$ from (7).

Thus, we conclude that $f^{\#}(0) \leq 3 K$ in all cases.

If $z_{0} \in D$, we may define $F(z)=f\left(\left(z+z_{0}\right) /\left(1+\bar{z}_{0} z\right)\right)$ and the previous argument shows that

$$
F^{\#}(0)=\left(1-\left|z_{0}\right|^{2}\right) f^{\#}\left(z_{0}\right) \leq 3 K
$$

for each $z_{0} \in D$. This completes the proof of Theorem 1 . 
We remark that if $p=2$ and $I<\pi$ then Lemma 1 shows that

$$
\left(1-|z|^{2}\right) f^{\#}(z) \leq \sqrt{\frac{I}{\pi-I}}
$$

since $I$ is an upper bound on the spherical area of $f(D)$. If $p=2$ and $\pi \leq I$, we can choose a number $K$ such that $S^{\prime} \leq 1$ in (5) and then define $\varrho$ by the equation

$$
\frac{\pi K^{2} \varrho^{2}}{1-\varrho^{2}}=1
$$

Then the method above yields that

$$
\left(1-|z|^{2}\right) f^{\#}(z) \leq\left\{2\left(1+\pi K^{2}\right)\right\}^{1 / 2} .
$$

However, $K$ may be arbitrarily large for bounded $I$. For instance, if $f(z)=k z$, where $0<k<\infty$, then $f^{\#}(0)=k$ while $I<\pi$. In particular, we see that the functions for which $I<\pi$, while individually normal, do not form a normal family. On the other hand, Theorem 1 shows that the automorphic functions satisfying the hypothesis of that theorem for an arbitrary group $\Gamma$, for a fixed $p$, where $p>2$, and for $I<I_{0}$, where $I_{0}$ is a fixed constant, do form a normal family. In Theorem 5 we will give an example to show that if $p<2$ then there exists a non-normal function which satisfies (2).

For a Fuchsian group $\Gamma$ and a positive number $p$, let $H_{a}(\Gamma, p)$ denote the set of all functions $f$ meromorphic in $D$ such that $f$ is automorphic with respect to $\Gamma$ and (2) is satisfied. Then we can obtain the following result.

Corollary 1. If $\Gamma$ is a Fuchsian group and $2 \leq p<q$, then $H_{a}(\Gamma, p) \subset$ $H_{a}(\Gamma, q)$. Further, if $m(F)<\infty$, then $H_{a}(\Gamma, p)=H_{a}(\Gamma, q)$.

Proof. Suppose that $f \in H_{a}(\Gamma, p)$ and that $2 \leq p<q$. By Theorem 1 , $f \in H_{a}(\Gamma, p)$ implies that $f$ is a normal function, so there exists a constant $C_{f}$ such that $\left(1-|z|^{2}\right) f^{\#}(z) \leq C_{f}$ for each $z \in D$. Then

$$
\begin{aligned}
\iint_{F} & {\left[\left(1-|z|^{2}\right) f^{\#}(z)\right]^{q} d m(z) } \\
& =\iint_{F}\left[\left(1-|z|^{2}\right) f^{\#}(z)\right]^{p}\left[\left(1-|z|^{2}\right) f^{\#}(z)\right]^{q-p} d m(z) \\
& \leq\left(C_{f}\right)^{q-p} \iint_{F}\left[\left(1-|z|^{2}\right) f^{\#}(z)\right]^{p} d m(z) .
\end{aligned}
$$

Thus $f \in H_{a}(\Gamma, q)$, which proves the first part of the Corollary.

Now suppose that $f \in H_{a}(\Gamma, q)$ and that $m(F)<\infty$. Then, if $A=\{z \in F$ : $\left.\left(1-|z|^{2}\right) f^{\#}(z)<1\right\}$, we have that

$$
\iint_{F}\left[\left(1-|z|^{2}\right) f^{\#}(z)\right]^{p} d m(z) \leq \iint_{F}\left[\left(1-|z|^{2}\right) f^{\#}(z)\right]^{q} d m(z)+m(A)<\infty,
$$

and it follows that $f \in H_{a}(\Gamma, p)$, which proves the second part of the Corollary. 
We will show by an example in Section 6 that, if $\Gamma$ is a group of rotations about the origin, then the classes $H_{a}(\Gamma, p)$, for $2 \leq p<\infty$, are all distinct. In this case, $F$ is a sector of the unit disk so that $m(F)=\infty$ and thus Corollary 1 does not apply. We do not know if $H_{a}(\Gamma, p)=H_{a}(\Gamma, q), 2 \leq p<q$, can happen for other Fuchsian groups $\Gamma$ for which $m(F)=\infty$.

Theorem 1 has implications for some rotation automorphic functions, as well as for automorphic functions.

Corollary 2. Let $f$ be a rotation automorphic function with respect to a Fuchsian group $\Gamma$ such that (2) is satisfied for $p \geq 2$. If the rotation group $\Sigma=\left\{S_{\gamma}: \gamma \in \Gamma\right\}$ is discrete, then $f$ is a normal function.

Proof. Define $\Gamma^{\prime}=\left\{\gamma \in \Gamma: S_{\gamma}=\right.$ identity $\}$. Then $\Gamma^{\prime}$ is a subgroup of $\Gamma$, and $f$ is an automorphic function relative to the Fuchsian group $\Gamma^{\prime}$. Since $\Sigma$ is a discrete group, there exist $n$ elements $\gamma_{0}=$ identity, $\gamma_{1}, \gamma_{2}, \ldots, \gamma_{n-1}$ in $\Gamma$ such that, for each $\gamma \in \Gamma$, there exists an element $\gamma^{\prime} \in \Gamma^{\prime}$ and an integer $j$, $0 \leq j \leq n-1$, such that both $\gamma=\gamma_{j} \circ \gamma^{\prime}$ and $S_{\gamma} \circ\left(S_{\gamma_{j}}^{-1}\right)=$ identity (see [5]). Let $F^{\prime}$ be the interior of the set $\cup_{j=0}^{n-1} \gamma_{j}(\bar{F})$. Then the union of $F^{\prime}$ and an appropriate subset of its boundary forms a fundamental set for the Fuchsian group $\Gamma^{\prime}$. Thus, applying (2), we get

$$
\iint_{F^{\prime}}\left(1-|z|^{2}\right)^{p-2}\left(f^{\#}(z)\right)^{p} d x d y \leq n \iint_{F}\left(1-|z|^{2}\right)^{p-2}\left(f^{\#}(z)\right)^{p} d x d y<\infty
$$

and it now follows from Theorem 1 that $f$ is a normal function.

We note that Corollary 2 generalizes a result of the first author [2, p. 372], where the result of the Corollary is given for the case $p=2$.

\section{Rotation automorphic functions}

In this section we prove two theorems relating condition (2) for rotation automorphic functions to normal functions. The first of these generalizes a result of the first author [1, 1.3 Theorem, p. 209].

Theorem 2. Let $\Gamma$ be a Fuchsian group such that $\Gamma$ consists of the identity and hyperbolic transformations and there is a constant $t_{0}>2$ such that $\mid$ trace $\gamma \mid \geq$ $t_{0}$ for all $\gamma \in \Gamma, \gamma \neq$ identity. Let $f$ be a rotation automorphic function with respect to $\Gamma$ which satisfies (2). Then $f$ is a normal function.

Proof. By Lemma 1, it suffices to show that there exist numbers $r_{1}$ and $\alpha$, where $r_{1}>0$ and $0<\alpha<\pi$, such that for each $z_{0} \in D$,

$$
\iint_{U\left(z_{0}, r_{1}\right)} f^{\#}(z)^{2} d x d y \leq \alpha
$$


where $U\left(z_{0}, r_{1}\right)$ denotes the hyperbolic disk

$$
U\left(z_{0}, r_{1}\right)=\left\{z \in D:\left|\left(z-z_{0}\right) /\left(1-\bar{z}_{0} z\right)\right|<r_{1}\right\}
$$

But, given $\alpha<\pi$, from condition (2) there exists a positive $\delta$ such that

$$
\iint_{A}\left(1-|z|^{2}\right)^{p-2}\left(f^{\#}(z)\right)^{p} d x d y=\iint_{A}\left[\left(1-|z|^{2}\right) f^{\#}(z)\right]^{p} d m(z)<\alpha
$$

whenever $A \subset F$ and $m(A)<\delta$, where $d m(z)=\left(1-|z|^{2}\right)^{-2} d x d y$ denotes the element of hyperbolic area. The hypotheses on the group $\Gamma$ guarantee that there exist a positive number $r_{1}$ and a positive integer $K$ such that, for each $z_{0} \in D$,

$$
n\left(U\left(z_{0}, r_{1}\right), z\right) \leq K
$$

for each $z \in D$, where $n\left(U\left(z_{0}, r_{1}\right), z\right)$ denotes the number of points in $U\left(z_{0}, r_{1}\right)$ equivalent under $\Gamma$ to $z$ (see [12, Lemma 1, p. 284]). It is no loss of generality to assume that $\alpha<\pi /(K+1)$ and that $r_{1}$ is so small that both $m\left(U\left(z_{0}, r_{1}\right)\right)<\alpha$ and $m\left(U\left(z_{0}, r_{1}\right)\right)<\delta$. Thus, $U\left(z_{0}, r_{1}\right)$ can be divided into $K$ regions $A_{\nu}, 1 \leq \nu \leq K$, each equivalent under $\Gamma$ to a subset $F_{\nu}$ of $F$. Also, each $F_{\nu}$ has measure less than $\delta$. Thus

$$
\begin{aligned}
\iint_{U\left(z_{0}, r_{1}\right)} & f^{\#}(z)^{2} d x d y=\iint_{U\left(z_{0}, r_{1}\right)}\left[\left(1-|z|^{2}\right) f^{\#}(z)\right]^{2} d m(z) \\
& \leq \iint_{U\left(z_{0}, r_{1}\right)}\left[\left(1-|z|^{2}\right) f^{\#}(z)\right]^{p} d m(z)+m\left(U\left(z_{0}, r_{1}\right)\right) \\
& \leq m\left(U\left(z_{0}, r_{1}\right)\right)+\sum_{\nu=1}^{K} \iint_{F_{\nu}}\left[\left(1-|z|^{2}\right) f^{\#}(z)\right]^{p} d m(z) \\
& \leq \alpha+K \alpha=(K+1) \alpha<\pi
\end{aligned}
$$

(Here we are using the fact that $x^{2} \leq x^{p}+1$ for $p \geq 2, x \geq 0$.) Thus, $f$ is a normal function.

We can also deal with the case that $\Gamma$ is a finitely generated group, even if $\Gamma$ has parabolic vertices.

Theorem 3. Let $f$ be a rotation automorphic function with respect to a finitely generated Fuchsian group $\Gamma$. If (2) is satisfied for some $p \geq 2$, then $f$ is a normal function and also $\lim \sup _{|z| \rightarrow 1, z \in F}\left(1-|z|^{2}\right) f^{\#}(z)=0$.

Proof. Since $\Gamma$ is finitely generated, the boundary of the fundamental region $F$ consists of a finite number of "sides" which are arcs of circles orthogonal to the 
unit circle, a finite number of free $\operatorname{arcs}$ on $\partial D$, and a finite number $q$ of parabolic vertices.

Let $W$ be a connected subset of the closure of $F$ such that the closure of $W$ does not contain a parabolic vertex, but $W$ contains a neighborhood of each point of the closure of each free arc of $\partial F \cap \partial D$. There exist a positive number $r$ and a positive integer $n_{1}$ such that, for each point $z_{0} \in W$, the disk $U\left(z_{0}, r\right)$ meets at most $n_{1}$ copies of $F$, including $F$ itself as one of these. Since $\left(1-|z|^{2}\right) f^{\#}(z)$ and $(d x d y) /\left(1-|z|^{2}\right)^{2}$ are invariant under $\Gamma$, it follows that we can choose $r$ sufficiently small so that

$$
\iint_{U\left(z_{0}, r\right)}\left(1-|z|^{2}\right)^{p-2}\left(f^{\#}(z)\right)^{p} d x d y<\pi n_{1} /\left(n_{1}+q_{1}\right)
$$

where $q_{1}=q+2$. As before, we have

$$
\iint_{U\left(z_{0}, r\right)}\left(f^{\#}(z)\right)^{2} d x d y \leq \iint_{U\left(z_{0}, r\right)}\left(1-|z|^{2}\right)^{p-2}\left(f^{\#}(z)\right)^{p} d x d y+m\left(U\left(z_{0}, r\right)\right) .
$$

It is no loss of generality to assume that $m\left(U\left(z_{0}, r\right)\right)<\pi /\left(n_{1}+q_{1}\right)$, and thus

$$
\iint_{U\left(z_{0}, r\right)}\left(f^{\#}(z)\right)^{2} d x d y<\left(n_{1}+1\right) \pi /\left(n_{1}+q_{1}\right)
$$

for $z_{0} \in W$.

Now suppose that $F$ has a parabolic vertex $p$. Let $\gamma_{p}$ be an element of $\Gamma$ such that $\gamma_{p}$ fixes $p, \gamma_{p} \neq$ identity, and $\gamma_{p}$ generates the subgroup of all parabolic elements of $\Gamma$ which fix $p$. There exists a rotation $S_{\gamma_{p}}$ of the Riemann sphere such that $f\left(\gamma_{p}(z)\right)=S_{\gamma_{p}}(f(z))$ for each $z \in D$. It is no loss of generality to assume that $S_{\gamma_{p}}(w)=e^{2 \pi \alpha i} w, 0<\alpha<1$, because if $\alpha=0$ then the desired result follows immediately from Theorem 1 . Let $c$ be a constant such that $i c p$ is a real number and

$$
1 /\left(\gamma_{p}(z)-p\right)=c+1 /(z-p)
$$

and set $t=\varphi(z)=\exp \{2 \pi i /(c(z-p))\}$. Then $t(z)$ maps a parabolic sector $\sigma_{p}$ in a one-to-one manner onto a set of the form $D^{*}=\{t: 0<|t|<\delta, d<\arg t<$ $2 \pi+d\}$. If we define $G(t)=f\left(\varphi^{-1}(t)\right)$ and define $H(t)=G(t) / t^{\alpha}$, we see that

$$
\iint_{\sigma_{p}}\left(f^{\#}(z)\right)^{2} d x d y=\iint_{D^{*}}\left(G^{\#}(t)\right)^{2} d x_{t} d y_{t}
$$

where $t=x_{t}+i y_{t}$. Further, since the hyperbolic area of the hyperbolic sector $\sigma_{p}$ is finite (we assume that $\sigma_{p}$ is restricted to a suitable neighborhood of $p$ ), we have

$$
\begin{aligned}
\iint_{\sigma_{p}}\left(f^{\#}(z)\right)^{2} d x d y & =\iint_{\sigma_{p}}\left[\left(1-|z|^{2}\right) f^{\#}(z)\right]^{2} d m(z) \\
& \leq \iint_{\sigma_{p}}\left[\left(1-|z|^{2}\right) f^{\#}(z)\right]^{p} d m(z)+m\left(\sigma_{p}\right)
\end{aligned}
$$


and so (2) implies that

$$
\iint_{D^{*}}\left(G^{\#}(t)\right)^{2} d x_{t} d y_{t}<\infty
$$

By a result in [3, 1.3 Lemma, p. 89], the function $H(t)$ is meromorphic at $t=0$. This means that either $G(t) \rightarrow 0$ or $G(t) \rightarrow \infty$ as $t \rightarrow 0$ in $D^{*}$, and this means that $f(z)$ has the limit either 0 or $\infty$ as $z \rightarrow p$ from within $F$. Thus, since $S_{\gamma_{p}}$ is a rotation about the origin (and $\infty$ ), it follows that $f(z)$ has the limit of either 0 or $\infty$ as $z \rightarrow p$ non-tangentially in $D$. For definiteness, assume that this limit is 0 . (We may replace $f$ by $1 / f$ without effecting any of our hypotheses.) It follows that we can choose the parabolic sector $\sigma_{p}$ and $r>0$ such that, if $z_{0} \in \sigma_{p}$ then, $|f(z)|<1$ for $z \in U\left(z_{0}, r\right)$. This implies that there exists a constant $K_{r}$, depending only on $r$, such that $\left|f^{\prime}(z)\right|\left(1-|z|^{2}\right)<K_{r}$ for $z \in U\left(z_{0}, r\right)$. Further, since $K_{r}$ decreases with $r$, we may choose $r>0$ such that

$$
\begin{aligned}
\iint_{U\left(z_{0}, r\right)}\left(f^{\#}(z)\right)^{2} d x d y & =\iint_{U\left(z_{0}, r\right)}\left[\left(1-|z|^{2}\right) f^{\#}(z)\right]^{2} d m(z) \\
& \leq\left(K_{r}^{2}\right) m\left(U\left(z_{0}, r\right)\right)<\pi /\left(n_{1}+q_{1}\right),
\end{aligned}
$$

where $n_{1}$ and $q_{1}$ are the numbers in (8).

For each of the finite number of parabolic vertices $p$, let $W_{p}$ be a parabolic sector contained in $\sigma_{p}$ such that (9) is satisfied whenever $z_{0} \in W_{p}$ and $r$ is chosen as above. Let $W=F-\cup_{p} W_{p}$. Combining the finite number of cases considered (a finite number of parabolic sectors plus the one region containing the remainder of $F$ ), and using (8) and (9), we have

$$
\iint_{U\left(z_{0}, r\right)}\left(f^{\#}(z)\right)^{2} d x d y \leq\left(n_{1}+q+1\right) \pi /\left(n_{1}+q_{1}\right)<\pi
$$

for each choice of $z_{0}$ in $F$. It follows that $f$ is a normal function in $D$.

Now suppose that $\left\{z_{n}\right\}$ is a sequence of points in $F$ such that $z_{n} \rightarrow \zeta$, where $\zeta \in \partial D$ and $\left(1-\left|z_{n}\right|^{2}\right) f^{\#}\left(z_{n}\right) \geq K>0$. We shall show that this assumption leads to a contradiction. We define

$$
f_{n}(w)=f\left(\frac{w+z_{n}}{1+\bar{z}_{n} w}\right)
$$

Then, since $f$ is a normal function in $D$, a subsequence of $\left\{f_{n}(w)\right\}$ converges uniformly on each compact subset of $D$ to a meromorphic function $f_{0}(w)$. We may replace the sequence $\left\{f_{n}(w)\right\}$ by this subsequence, and so assume, without loss of generality, that the sequence $\left\{f_{n}(w)\right\}$ converges to $f_{0}(w)$ uniformly on each compact subset of $D$. Also,

$$
f_{0}^{\#}(0)=\lim _{n \rightarrow \infty} f_{n}^{\#}(0)=\lim _{n \rightarrow \infty}\left(1-\left|z_{n}\right|^{2}\right) f^{\#}\left(z_{n}\right) \geq K .
$$


Now fix $r>0$ and define $D_{r}=\{w:|w| \leq \tanh (2 r)\}$. For $w \in D_{r}$, we have $f_{n}^{\#}(w) \rightarrow f_{0}^{\#}(w)$ and

$$
\begin{aligned}
\iint_{D_{r}}\left(1-|w|^{2}\right)^{p-2}\left(f_{n}^{\#}(w)\right)^{p} d u d v & =\iint_{D_{r}}\left[\left(1-|w|^{2}\right) f_{n}^{\#}(w)\right]^{p} d m(w) \\
& =\iint_{U\left(z_{n}, r\right)}\left[\left(1-|z|^{2}\right) f^{\#}(z)\right]^{p} d m(z) \\
& =\iint_{U\left(z_{n}, r\right)}\left(1-|z|^{2}\right)^{p-2}\left(f^{\#}(z)\right)^{p} d x d y
\end{aligned}
$$

where $w=u+i v$. If $\zeta$ is a point of a free $\operatorname{arc}$ of $\partial F \cap \partial D$, then $U\left(z_{n}, r\right)$ can meet at most two copies of $F$ for $n$ large, and then condition (2) implies that

$$
\iint_{D_{r}}\left(1-|w|^{2}\right)^{p-2}\left(f_{n}^{\#}(w)\right)^{p} d u d v \rightarrow 0
$$

It follows that $f_{0}^{\#}(0)=0$, contrary to hypothesis. If $\zeta$ is a parabolic vertex, the argument given to show that $f$ is normal again shows that $f_{0}^{\#}(0)=0$. This contradiction proves that

$$
\left(1-\left|z_{n}\right|^{2}\right) f^{\#}\left(z_{n}\right) \rightarrow 0
$$

as $z_{n} \rightarrow \partial D$ in $F$ and this completes the proof of Theorem 3 .

\section{Some cases where $p<2$}

For $f$ a meromorphic function in $D$, we define

$$
L(r)=\int_{0}^{2 \pi} f^{\#}\left(r e^{i \theta}\right) r d \theta
$$

We say that a meromorphic function $f$ is in the class $T_{1}(l)$ if $\lim _{\sup _{r \rightarrow 1}} L(r)=$ $\lambda \leq l$. We define $T_{1}=\cup_{0 \leq l<\infty} T_{1}(l)$. For a Jordan curve $\gamma$ in $D$, we define

$$
L(\gamma)=\int_{\gamma} f^{\#}(z)|d z|
$$

We say that the meromorphic function $f$ is in $T_{2}(l)$ if there exists a sequence of Jordan domains $\left\{D_{n}\right\}$ such that $\cup_{n=1}^{\infty} D_{n}=D$,

$$
\bar{D}_{n} \subset D_{n+1} \subset \bar{D}_{n+1} \subset D
$$

for each $n$, and $\lim \sup _{n \rightarrow \infty} L\left(\partial D_{n}\right)=\Lambda \leq l$. Finally, we define $T_{2}=$ $\cup_{0 \leq l<\infty} T_{2}(l)$. We remark that $T_{1}(l) \subset T_{2}(l)$ for each $l \geq 0$ and that $T_{1} \subset T_{2}$. The following theorem summarizes some results which the second author proved in [8] and [9]. 
Theorem H. (i) There exists a function $f$ which is not a normal function in $D$ but $f \in T_{1}[8$, Theorem 1 , p. 186].

(ii) If $f \in T_{1}(l)$ with $l<\pi$, then $f$ is a normal function in $D$. Thus, if $f \in T_{1}(0)$ then $f$ is a constant function [9, Theorem 4, p. 5].

(iii) There exists a non-constant analytic function in $T_{2}(0)[9$, Theorem 5, p. 5].

(iv) If $f$ is a function in $T_{2}$ and $\zeta \in \partial D$, then either

$$
\limsup _{z \rightarrow \zeta}\left(1-|z|^{2}\right) f^{\#}(z)=\infty
$$

or $f$ has a continuous extension to an open arc of $\partial D$ containing $\zeta$. In particular, if $f \in T_{2}$ and $f$ is a normal function in $D$, then $f$ has a continuous extension to the closure of $D$ [9, Theorem 1, p. 3].

Here, we prove the following result.

Theorem 4. If $f$ is a function meromorphic in $D$, and if

$$
\iint_{D}\left(1-|z|^{2}\right)^{-1}\left(f^{\#}(z)\right)^{s} d x d y<\infty
$$

where $s \geq 1$, then $f$ is either a constant function, or else $f$ satisfies (10) at each point $\zeta \in \partial D$. If $s \geq 2$, then $f$ is a constant function.

Proof. Condition (11) implies that

$$
\int_{0}^{2 \pi} \int_{r}^{(1+r) / 2}\left(1-|z|^{2}\right)^{-1}\left(f^{\#}(z)\right)^{s} r d r d \theta \rightarrow 0
$$

as $r \rightarrow 1$, which, in turn, implies that

$$
\Lambda_{r}=\inf \{L(t): r \leq t \leq(1+r) / 2\} \rightarrow 0 .
$$

This means that $f \in T_{2}(0)$. The first part of the theorem now follows from part (iv) of Theorem $\mathrm{H}$, for a continuous extension of $f$ to an arc of $\partial D$, combined with the condition that

$$
\liminf _{t \rightarrow 1} L(t)=0
$$

means that $f$ is constant on an $\operatorname{arc}$ of $\partial D$, and this means that $f$ is a constant function.

If $s \geq 2$, then

$$
\iint_{D}\left(1-|z|^{2}\right)^{s-2}\left(f^{\#}(z)\right)^{s} d x d y \leq \iint_{D}\left(1-|z|^{2}\right)^{-1}\left(f^{\#}(z)\right)^{s} d x d y<\infty,
$$

by (11) and now Theorem 1 , with $D=F$ and $\Gamma=$ identity , implies that $f$ is normal, so (10) cannot hold. Thus $f$ is constant in this case. This completes the proof of the Theorem. 


\section{An example to illustrate Theorem 4}

In this section we prove that functions considered in Theorem 4 actually exist.

Theorem 5. Suppose that $0<s<2$ and let $k(r)$ be a positive continuous function in $[0,1)$. Then there exists a non-constant function $f(z)$ analytic in $D$ such that

$$
\int_{0}^{2 \pi} \int_{0}^{1} k(r)\left(f^{\#}\left(r e^{i \theta}\right)\right)^{s} r d r d \theta<\infty
$$

Of course, we have in mind the situation for which $k(r)=(1-r)^{\beta}$, where $\beta$ is a suitable negative number. The case $s=1$ and $\beta=-1$ is a natural case of interest. This case also gives a clear contrast with the result of Metzger and Rao [15, Theorem 3] stated in Section 1. Since the general function $k(r)$ does not cause undue complications in the proof, we give the proof in the general case.

The proof of the Theorem is lengthy, and we will proceed in stages with several parts. First, we set up some of the constants and notation, and make some preliminary estimates. Then, we will prove three Lemmas. And finally, in the last part of the proof we give an estimate for the integral in (12).

To begin the proof, we define $\sigma=\min \{s / 2,1 / 2-s / 4\}$. We will assume throughout that $k(r)$ is a strictly increasing function such that $k(r) \rightarrow \infty$ as $r \rightarrow 1-$, for if it is not we can replace it by

$$
K(r)=(1-r)^{-1}+\sup \{k(t): 0 \leq t \leq r\} .
$$

Let $N$ be the least positive integer such that $\exp \left(2^{N-3} \sigma\right) \geq k(1 / 2)$ and, for $n \geq N$, let $R_{n}$ be the solution to the equation

$$
k\left(R_{n}\right)=\exp \left(2^{n-3} \sigma\right) .
$$

Clearly, we have $1 / 2 \leq R_{n}<R_{n+1}<1$ for $n \geq N$ and $R_{n} \rightarrow 1$ as $n \rightarrow \infty$. Let us define $m_{N}$ to be the least positive integer satisfying both $2^{-\left(m_{N}+1\right)}<R_{N+1}-R_{N}$ and $m_{N} \geq N$. We define

$$
r_{k}=(1 / 2)-(1 / 2)^{k+1}, \quad 0 \leq k \leq m_{N} .
$$

This has the properties that $r_{m_{N}}<\frac{1}{2} \leq R_{N}$ and $r_{m_{N}}-r_{m_{N}-1}<R_{N+1}-R_{N}$. We can now define the rest of the sequence $\left\{r_{n}\right\}$ inductively.

Suppose that we have defined a strictly increasing sequence of positive integers $m_{N}, m_{N+1}, \ldots, m_{j}$ with $j \geq N$, together with a strictly increasing sequence of numbers $r_{k}, 0 \leq k \leq m_{j}$, such that $r_{m_{j}}<R_{j}, r_{m_{j}}-r_{m_{j}-1}<R_{j+1}-R_{j}$, and $r_{k}-r_{k-1} \leq r_{k-1}-r_{k-2}$ for $0 \leq k \leq m_{j}$. Let $p_{j}$ be the least non-negative integer such that

$$
r_{m_{j}}+p_{j}\left(r_{m_{j}}-r_{m_{j}-1}\right)<R_{j} .
$$


(It is possible that $p_{j}=0$.) If $p_{j}>0$, then for $m_{j}<k \leq m_{j}+p_{j}$ we define $r_{k}$ so that $r_{k}-r_{k-1}=r_{k-1}-r_{k-2}$. Let $q_{j}$ be the least positive integer such that

$$
2^{-q_{j}}\left(r_{m_{j}}-r_{m_{j}-1}\right)<R_{j+2}-R_{j+1} \text {. }
$$

We define $m_{j+1}=m_{j}+p_{j}+q_{j}$, and for $m_{j}+p_{j}<k \leq m_{j+1}$ we define $r_{k}$ such that $r_{k}-r_{k-1}=\left(r_{k-1}-r_{k-2}\right) / 2$.

Our choices of $p_{j}$ and $q_{j}$ yield that $r_{m_{j}+1}<R_{j+1}$ and $r_{m_{j+1}}-r_{m_{j+1}-1}<$ $R_{j+2}-R_{j+1}$, which brings us full circle in the construction. Thus, sequences $\left\{m_{j}: j \geq N\right\}$ and $\left\{r_{k}\right\}$ can be constructed so that $r_{m_{j}}<R_{j}$ and so $r_{j}<R_{j}$ for $j \geq N$. Also,

$$
\left(r_{k-1}-r_{k-2}\right) / 2 \leq r_{k}-r_{k-1} \leq r_{k-1}-r_{k-2}
$$

and so

$$
2^{-(k+1)} \leq r_{k}-r_{k-1} \leq r_{k-1}-r_{k-2}, \quad k \geq 1 .
$$

Our construction also ensures that

$$
r_{m_{j}+p_{j}}<R_{j}<r_{m_{j}+p_{j}}+r_{m_{j}}-r_{m_{j}-1} .
$$

Thus, $r_{k} \rightarrow 1$ as $k \rightarrow \infty$, since $R_{j} \rightarrow 1$ as $j \rightarrow \infty$.

We now define, for $n \geq 0$,

$$
P_{n}=4^{n+2}, \quad r_{n}^{\prime}=\left(r_{n}+r_{n+1}\right) / 2,
$$

and, for $n \geq 1$,

$$
E_{n}=\left\{z: r_{n-1}^{\prime} \leq|z| \leq r_{n}^{\prime}\right\}
$$

and

$$
G_{n}(z)=1+\left(z / r_{n}\right)^{P_{n}}, \quad \text { and } \quad g_{n}(z)=\prod_{k=1, k \neq n}^{\infty} G_{k}(z)
$$

and

$$
f(z)=\prod_{n=1}^{\infty} G_{n}(z)
$$

so that $f(z)=g_{n}(z) G_{n}(z), n \geq 1$.

Lemma 2. We define

$$
B=(4 / 3)+7 \sum_{k=1}^{\infty} \exp \left(-\left(\frac{1}{2} k^{1 / 2}\right)\right)
$$


and, for $n \geq 1$,

$$
A_{n}=\prod_{j=n+1}^{\infty}\left(1-2^{-(j+2)}\right)
$$

and

$$
M_{n}=A_{n} \exp \left\{\sum_{j=1}^{n-1} P_{j}\left(r_{n}-r_{j}\right) / 4\right\} .
$$

Then, for $n \geq 3$ and $z \in E_{n}$, the following are valid:

(i) $M_{n}<\left|g_{n}(z)\right|<\left(M_{n}\right)^{30}$,

(ii) $M_{n}>\exp \left(2^{n-1}\right)>2^{n-1}$, and

(iii) $\left|g_{n}^{\prime}(z) / g_{n}(z)\right| \leq B P_{n}$.

Proof. For $1 \leq j<n$ and $z \in E_{n}$,

$$
\left(\frac{r_{n-1}^{\prime}}{r_{j}}\right)^{P_{j}}-1 \leq\left|G_{j}(z)\right| \leq\left(\frac{r_{n}^{\prime}}{r_{j}}\right)^{P_{j}}+1 .
$$

By (14) we have that

$$
\begin{aligned}
\left(\frac{r_{n-1}^{\prime}}{r_{j}}\right)^{P_{j}} & =\left(1+\frac{r_{n-1}^{\prime}-r_{j}}{r_{j}}\right)^{P_{j}} \geq\left(1+\frac{r_{n}-r_{j}}{2}\right)^{P_{j}} \\
& \geq \exp \left(\frac{P_{j}\left(r_{n}-r_{j}\right)}{3}\right) \geq e^{8 / 3}>14 .
\end{aligned}
$$

Thus,

$$
\left|G_{j}(z)\right|>\exp \left(\frac{P_{j}\left(r_{n}-r_{j}\right)}{3}\right)-1>\exp \left(\frac{P_{j}\left(r_{n}-r_{j}\right)}{4}\right) .
$$

Also, using (13), we deduce that $r_{j} \geq 1 / 4$ and

$$
r_{n}^{\prime}-r_{j}=\left(r_{n}^{\prime}-r_{n}\right)+\left(r_{n}-r_{j}\right) \leq \frac{1}{2}\left(r_{n}-r_{j}\right)+\left(r_{n}-r_{j}\right)=\frac{3}{2}\left(r_{n}-r_{j}\right),
$$

yielding that

$$
\begin{aligned}
1+\left(\frac{r_{n}^{\prime}}{r_{j}}\right)^{P_{j}} & \leq 2\left(\frac{r_{n}^{\prime}}{r_{j}}\right)^{P_{j}}=2\left(1+\frac{r_{n}^{\prime}-r_{j}}{r_{j}}\right)^{P_{j}} \\
& \leq 2\left(1+6\left(r_{n}-r_{j}\right)\right)^{P_{j}}<2 \exp \left(6 P_{j}\left(r_{n}-r_{j}\right)\right)<\exp \left(7 P_{j}\left(r_{n}-r_{j}\right)\right) .
\end{aligned}
$$

Now, for $j>n$,

$$
\begin{aligned}
\left|\frac{z}{r_{j}}\right|^{-P_{j}} & \geq\left(\frac{r_{j}}{r_{n}^{\prime}}\right)^{P_{j}}=\left(1+\frac{r_{j}-r_{n}^{\prime}}{r_{n}^{\prime}}\right)^{P_{j}} \\
& \geq\left(1+\frac{r_{n+1}-r_{n}}{2}\right)^{P_{j}} \geq \exp \left\{\frac{P_{j}\left(r_{n+1}-r_{n}\right)}{3}\right\} .
\end{aligned}
$$


Hence, for $j>n$ we have $j+1 \geq n+2$, so, using (14) again, we deduce that

$$
\left|\frac{z}{r_{j}}\right|^{-P_{j}} \geq \exp \left(4^{j+2} 2^{-j-1} / 3\right)=\exp \left(2^{j+3} / 3\right)>\exp \left(2^{j+1}\right)>2^{j+2}
$$

and thus,

$$
A_{n}=\prod_{j=n+1}^{\infty}\left(1-2^{-j-2}\right) \leq \prod_{j=n+1}^{\infty}\left|G_{j}(z)\right| \leq \prod_{j=n+1}^{\infty}\left(1+2^{-j-2}\right)<1 / A_{n}
$$

Thus,

$$
A_{n} \exp \left\{\sum_{j=1}^{n-1} P_{j}\left(r_{n}-r_{j}\right) / 4\right\} \leq\left|g_{n}(z)\right| \leq \frac{1}{A_{n}} \exp \left\{\sum_{j=1}^{n-1} 7 P_{j}\left(r_{n}-r_{j}\right)\right\} .
$$

Note that $A_{n}>\exp \left(-\frac{1}{4}\right)$, so $1 / A_{n}<A_{n}^{30} \exp (31 / 4)$. Hence,

$$
\left(1 / A_{n}\right) \exp \left\{\sum_{j=1}^{n-1} 7 P_{j}\left(r_{n}-r_{j}\right)\right\} \leq\left[A_{n} \exp \left\{\sum_{j=1}^{n-1} P_{j}\left(r_{n}-r_{j}\right) / 4\right\}\right]^{30}
$$

provided that $\sum_{j=1}^{n-1} P_{j}\left(r_{n}-r_{j}\right) / 2 \geq 31 / 4$, and this is certainly true for $n \geq 3$ since then $P_{n-1}\left(r_{n}-r_{n-1}\right) \geq 2^{n+1} \geq 16$. This proves (i) of Lemma 2 .

Also, we deduce using (14) that, for $n \geq 3$,

$$
\begin{aligned}
M_{n} & \geq \exp \left(-1 / 4+\sum_{j=1}^{n-1} P_{j}\left(r_{n}-r_{j}\right) / 4\right) \geq \exp \left(2^{n-1}-1 / 4+P_{n-2}\left(r_{n}-r_{n-2}\right)\right) \\
& \geq \exp \left(2^{n-1}-1 / 4+3(4)^{n-1}(2)^{-n-2}\right) \geq \exp \left(2^{n-1}+1 / 8\right)
\end{aligned}
$$

This proves (ii).

To prove (iii), we have

$$
\frac{g_{n}^{\prime}(z)}{g_{n}(z)}=\sum_{j=1}^{n-1} \frac{\left(P_{j} / r_{j}\right)\left(z / r_{j}\right)^{P_{j}-1}}{1+\left(z / r_{j}\right)^{P_{j}}}+\sum_{j=n+1}^{\infty} \frac{\left(P_{j} / r_{j}\right)\left(z / r_{j}\right)^{P_{j}-1}}{1+\left(z / r_{j}\right)^{P_{j}}}
$$

If $j<n$, then $\left|z / r_{j}\right|^{P_{j}}>14$ by (15) and, since $n \geq 2,|z|>r_{1}^{\prime} \geq 5 / 16$, and thus

$$
\begin{aligned}
& \sum_{j=1}^{n-1} \frac{\left(P_{j} / r_{j}\right)\left|z / r_{j}\right|^{P_{j}-1}}{1+\left|z / r_{j}\right|^{P_{j}}} \leq \sum_{j=1}^{n-1} \frac{\left|P_{j}\right|}{|z|}\left(\frac{\left|z / r_{j}\right|^{P_{j}}}{\left|z / r_{j}\right|^{P_{j}}-1}\right) \\
& \quad<\sum_{j=1}^{n-1}(16 / 5)(14 / 13) P_{j}<4 \sum_{j=1}^{n-1} P_{j}=4 \sum_{j=1}^{n-1} 4^{j+2}<(4 / 3) P_{n}
\end{aligned}
$$


Now, for $j>n$, we have from (16) that

$$
\left|z / r_{j}\right|^{P_{j}} \leq \exp \left(-2^{j+1}\right)=\exp \left\{-\left(P_{j}^{1 / 2}\right) / 2\right\}<\frac{1}{2} .
$$

Thus,

$$
\begin{aligned}
& \sum_{j=n+1}^{\infty} \frac{\left(P_{j} / r_{j}\right)\left|z / r_{j}\right|^{P_{j}-1}}{1+\left|z / r_{j}\right|^{P_{j}}} \leq \frac{2}{|z|} \sum_{j=n+1}^{\infty} P_{j}\left|z / r_{j}\right|^{P_{j}} \\
& \quad \leq(32 / 5) \sum_{j=n+1}^{\infty} P_{j} \exp \left\{-\frac{1}{2} P_{j}^{1 / 2}\right\} \leq 7 \sum_{j=1}^{\infty} j \exp \left\{-\frac{1}{2} j^{1 / 2}\right\}=A
\end{aligned}
$$

Hence, for $n \geq 2$,

$$
\left|g_{n}^{\prime}(z) / g_{n}(z)\right| \leq(4 / 3) P_{n}+A \leq(A+4 / 3) P_{n}=B P_{n},
$$

and this gives Lemma 2 (iii) and completes the proof of Lemma 2.

Lemma 3. Let $\delta$ be a fixed number satisfying $0<\delta<1 / 2$. For fixed $j$, $j \geq 1$, and $1 \leq k \leq P_{j}$, we define

$$
z_{k}=r_{j} \exp \left\{(2 k+1) \pi i / P_{j}\right\}
$$

and

$$
D_{k}=\left\{z:\left|z-z_{k}\right|<3 r_{j} \delta / P_{j}\right\}
$$

Then

$$
\left|G_{j}(z)\right|>\delta \quad \text { for } \quad z \in\{z:|z|<1\}-\bigcup_{k=1}^{P_{j}} D_{k} .
$$

Proof. Assume that $z$ is in $D$ and $\left|G_{j}(z)\right| \leq \delta$. Since

$$
1+\left(z / r_{j}\right)^{P_{j}}=\prod_{k=1}^{P_{j}}\left(z_{k}-z\right) / z_{k}
$$

we may assume, by symmetry, that $z$ is nearest to $z_{1}$. Then $\left|1+\left(z / r_{j}\right)^{P_{j}}\right| \leq \delta$ means that $\left(z / r_{j}\right)^{P_{j}}=-1+r \delta e^{i \theta}$, where $0 \leq r \leq 1$ and $0 \leq \theta \leq 2 \pi$. It follows that $z / r_{j}$ is a $P_{j}$-th root of $-1+r \delta e^{i \theta}$, which means that $|z| / r_{j}$ is a $P_{j}$-th root of $\left|-1+r \delta e^{i \theta}\right|$, so $1-\delta \leq\left|z / r_{j}\right|^{P_{j}} \leq 1+\delta$. A consequence of this is $1-\left(2 \delta / P_{j}\right)<\left|z / r_{j}\right|<1+\left(2 \delta / P_{j}\right)$ and, by considering arguments, we get

$$
(\pi-\arctan \delta) / P_{j}<\arg z<(\pi+\arctan \delta) / P_{j} .
$$


Thus, $z$ must lie in the set

$$
\begin{gathered}
\left\{z: 1-\left(2 \delta / P_{j}\right)<\left|z / r_{j}\right|<1+\left(2 \delta / P_{j}\right),\right. \\
\left.(\pi-\arctan \delta) / P_{j}<\arg z<(\pi+\arctan \delta) / P_{j}\right\} .
\end{gathered}
$$

This region is a sector of an annulus containing $z_{1} / r_{j}$, and we can compute the maximum distance of a point $w=z / r_{j}$ in this region from $z_{1} / r_{j}$ by moving first along $|z|=r_{j}$ and then radially out from the origin. This calculation gives

$$
\left|\left(z / r_{j}\right)-\left(z_{1} / r_{j}\right)\right| \leq 2 \delta / P_{j}+(\arctan \delta) / P_{j}<3 \delta / P_{j} .
$$

Thus, $\left|z-z_{1}\right|<3 \delta r_{j} / P_{j}$, and Lemma 3 is proved.

Lemma 4. If $0<s<2, \sigma=\min \left\{\frac{1}{2} s, \frac{1}{2}-\frac{1}{4} s\right\}, n>n_{0}$, then

$$
\iint_{E_{n}}\left(f^{\#}(z)\right)^{s} d x d y<W M_{n}^{-\sigma}=O\left(\exp \left(-2^{n-1} \sigma\right)\right),
$$

where $W$ is a constant independent of $n$.

Proof. We define $E_{n, 0}=\left\{z: z \in E_{n}\right.$ and $\left.\left|G_{n}(z)\right|>1 / 2\right\}$ and assume that $n \geq 3$ and $z \in E_{n, 0}$. Then, by (13), $|z| \geq\left(r_{0}+r_{1}\right) / 2=1 / 8$, since $n \geq 1$, so

$$
\left|G_{n}^{\prime}(z) / G_{n}(z)\right|=\left|P_{n} / z\right|\left|z / r_{n}\right|^{P_{n}} /\left|1+\left(z / r_{n}\right)^{P_{n}}\right| \leq 3 P_{n} /|z| \leq 24 P_{n} .
$$

Using this estimate and the result (iii) of Lemma 2, we have

$$
\left|f^{\prime}(z) / f(z)\right| \leq\left|G_{n}^{\prime}(z) / G_{n}(z)\right|+\left|g_{n}^{\prime}(z) / g_{n}(z)\right| \leq(24+B) P_{n}
$$

and also, $|f(z)|=\left|G_{n}(z) g_{n}(z)\right|>M_{n} / 2$ from (i) of Lemma 2. Thus,

$$
\begin{aligned}
f^{\#}(z) & <\left|f^{\prime}(z)\right| /|f(z)|^{2}=\left(\left|f^{\prime}(z) / f(z)\right|\right)(1 /|f(z)|)<2(24+B) P_{n} / M_{n} \\
& =2^{2 n+4}(48+2 B) / M_{n}=2^{6}\left(2^{n-1}\right)^{2}(48+2 B) / M_{n}<C\left(\log M_{n}\right)^{2} / M_{n}
\end{aligned}
$$

where $C=2^{6}(48+2 B)$ and the estimate $2^{n-1}<\log M_{n}$ is contained in Lemma 2 (ii). The area of $E_{n}$ is less than $\pi$, so

$$
\iint_{E_{n, 0}}\left(f^{\#}(z)\right)^{s} d x d y<\pi C^{s}\left(\log M_{n}\right)^{2 s} /\left(M_{n}\right)^{s}
$$

Next, we define

$$
E_{n, q}=\left\{z \in E_{n}: 2^{-q-1}<\left|G_{n}(z)\right| \leq 2^{-q}\right\}
$$


for $q \geq 1$. Assume that $z \in E_{n, q}$. Again we have that both $\left|z / r_{n}\right|$ and $\left|z / r_{n}\right|^{P_{n}}$ lie between $1 / 2$ and 2 , so

$$
\begin{aligned}
\left|G_{n}^{\prime}(z) / G_{n}(z)\right| & \leq 2^{q+1}\left|G_{n}^{\prime}(z)\right|=2^{q+1}\left|P_{n} / z\right|\left|z / r_{n}\right|^{P_{n}} \\
& \leq 2^{q+1}\left(P_{n} /|z|\right) 2<2^{q+5} P_{n} \quad(\text { since }|z| \geq 1 / 8)
\end{aligned}
$$

and hence by the same reasoning as before,

$$
\left|f^{\prime}(z) / f(z)\right|<2^{q+5} P_{n}+B P_{n} \leq 2^{q} F P_{n},
$$

where $F$ is a constant independent of $q$ or $n$. Thus,

$$
\begin{aligned}
f^{\#}(z) & \leq\left|f^{\prime}(z) /(f(z))^{2}\right|=\left(\left|f^{\prime}(z) / f(z)\right|\right)(1 /|f(z)|) \\
& <2^{q} F P_{n} /\left(2^{-q-1} M_{n}\right)=(2 F)\left(4^{q}\right) P_{n} / M_{n}<\left(2^{7} F\right)\left(4^{q}\right)\left(\log M_{n}\right)^{2} / M_{n} .
\end{aligned}
$$

Since $\left|G_{n}(z)\right| \leq 2^{-q}$, we can use Lemma 3 to conclude that the area of $E_{n, q}$ is not more than $P_{n} \pi\left(3 r_{n} /\left(2^{q} P_{n}\right)\right)^{2}<9 \pi /\left(4^{q} P_{n}\right)$. Thus,

$$
\begin{aligned}
\iint_{E_{n, q}}\left(f^{\#}(z)\right)^{s} d x d y & <\left\{\left(2^{7} F\right)\left(4^{q}\right)\left(\log M_{n}\right)^{2} / M_{n}\right\}^{s} 9 \pi /\left(4^{q} P_{n}\right) \\
& =4^{q(s-1)} V\left(\log M_{n}\right)^{2 s} /\left(M_{n}^{s} P_{n}\right),
\end{aligned}
$$

where $V$ is a constant independent of $n$ and $q$. Let $Q_{1}$ be the largest integer such that $4^{Q_{1}} \leq M_{n}$. Thus $Q_{1}<\log M_{n}$. Also, if $h=\max (0, s-1)$, we have that $4^{q(s-1)} \leq M_{n}^{h}, 1 \leq q \leq Q_{1}$. Thus

$$
\begin{aligned}
\sum_{q=1}^{Q_{1}} \iint_{E_{n, q}} & \left(f^{\#}(z)\right)^{s} d x d y \leq V Q_{1} M_{n}^{h}\left(\log M_{n}\right)^{2 s} /\left(M_{n}^{s} P_{n}\right) \\
& \leq V\left(\log M_{n}\right)^{2 s+1} M_{n}^{h-s} \leq V\left(\log M_{n}\right)^{2 s+1} M_{n}^{-2 \sigma} .
\end{aligned}
$$

We note that, by Lemma 2 (i), $|f(z)|<M_{n}^{30}$ for $z \in E_{n}$. Let $Q_{2}$ be the smallest integer such that $2^{Q_{2}}>M_{n}^{30}$. Then, for $Q_{1}<q \leq Q_{2}$, we have $f^{\#}(z) \leq$ $\left|f^{\prime}(z) / f(z)\right|<2^{q} F P_{n}$ from (17). (Note that $1 /\left(1+x^{2}\right)<1 / x$ for all positive $x$.) It follows that

$$
\iint_{E_{n, q}}\left(f^{\#}(z)\right)^{s} d x d y<\left(2^{q} F P_{n}\right)^{s}(9 \pi) /\left(4^{q} P_{n}\right),
$$

and thus

$$
\begin{aligned}
\sum_{q=Q_{1}+1}^{Q_{2}} & \iint_{E_{n, q}}\left(f^{\#}(z)\right)^{s} d x d y<\sum_{q=Q_{1}+1}^{Q_{2}} 9 \pi F^{s} P_{n}^{s-1} /\left(2^{q}\right)^{2-s} \\
& <\left(9 \pi F^{s}\right)\left(Q_{2}-1\right) P_{n}^{s-1} /\left(2^{Q_{1}}\right)^{2-s} \leq R\left(\log M_{n}\right) P_{n}^{s-1} / M_{n}^{(1-s / 2)} \\
& \leq R\left(\log M_{n}\right)\left(\log M_{n}\right)^{2 h} / M_{n}^{(1-s / 2)}
\end{aligned}
$$


where $R$ is a constant independent of $n$. (We use here the fact that $2^{Q_{1}} \leq M_{n}^{1 / 2}$ and that $\left(Q_{2}-1\right) \log 2 \leq 30 \log M_{n}$ from the way that $Q_{1}$ and $Q_{2}$ are defined.)

For $q>Q_{2}$, we have

$$
\begin{aligned}
f^{\#}(z) & \leq\left|f^{\prime}(z)\right|=\left|g_{n}^{\prime}(z) G_{n}(z)+g_{n}(z) G_{n}^{\prime}(z)\right| \\
& \leq\left|g_{n}(z)\right|\left\{\left|G_{n}^{\prime}(z)\right|+\left|G_{n}(z)\right|\left|g_{n}^{\prime}(z) / g_{n}(z)\right|\right\} \\
& \leq M_{n}^{30}\left\{\left(P_{n} /|z|\right)\left|z / r_{n}\right|^{P_{n}}+2 B P_{n}\right\} \leq M_{n}^{30} P_{n}\{16+2 B\} .
\end{aligned}
$$

Here we use Lemma 2 (iii) and the basic estimates from the earlier part of the proof of Lemma 4 . We now define

$$
E_{n}^{\prime}=E_{n}-\bigcup_{q=0}^{Q_{2}} E_{n, q}
$$

Then the area of $E_{n}^{\prime}$ is less than

$$
9 P_{n} \pi\left(2^{-Q_{2}} / P_{n}\right)^{2}=9 \pi 2^{-2 Q_{2}} / P_{n},
$$

and

$$
\begin{aligned}
\iint_{E_{n}^{\prime}}\left(f^{\#}(z)\right)^{s} d x d y & \leq\left(M_{n}^{30} P_{n}\{16+2 B\}\right)^{s}(9 \pi) 2^{-2 Q_{2}} / P_{n} \\
& <9 \pi\{16+2 B\}^{s}\left(M_{n}^{30}\right)^{s-2} P_{n}^{s-1}<L\left(\log M_{n}\right)^{2 h} /\left(M_{n}^{30}\right)^{2-s}
\end{aligned}
$$

where $L$ is a constant independent of $n$ and $Q_{2}$.

Combining all these four estimates, we obtain

$$
\iint_{E_{n}}\left(f^{\#}(z)\right)^{s} d x d y \leq W \max \left\{M_{n}^{-s / 2}, M_{n}^{-(1-s / 2) / 2}\right\}=W M_{n}^{-\sigma},
$$

where $W$ is a constant independent of $n$. (In each case, we used estimates of the form $(\log x)^{p} / x^{q}<A / x^{q / 2}$, where $A$ is a constant depending only on $p$ and $q$ for $x \geq 1$.) This completes the proof of Lemma 4 .

To complete the proof of Theorem 5, we give an upper bound for the integral in (12). We can express the unit disk $D$ by

$$
D=\{z:|z|<1\}=\{z:|z|<1 / 8\} \bigcup \bigcup_{n=1}^{\infty} E_{n}
$$

Hence, the integral in (12) is equal to

$$
\int_{0}^{2 \pi} \int_{0}^{1 / 8} k(r)\left(f^{\#}(z)\right)^{s} r d r d \theta+\sum_{n=1}^{\infty} \iint_{E_{n}} k(r)\left(f^{\#}(z)\right)^{s} r d r d \theta .
$$


For $n>n_{0}$ and $z \in E_{n}$, we have $k(|z|) \leq k\left(r_{n+1}\right)<k\left(R_{n+1}\right)=\exp \left\{2^{n-2} \sigma\right\}$. Thus,

$$
\begin{aligned}
\iint_{E_{n}} k(r)\left(f^{\#}(z)\right)^{s} r d r d \theta \leq \exp \left\{2^{n-2} \sigma\right\} W / M_{n}^{\sigma} \\
\quad \leq W \exp \left\{\left(2^{n-2}-2^{n-1}\right) \sigma\right\}=W \exp \left\{-2^{n-2} \sigma\right\} .
\end{aligned}
$$

Since $\sum \exp \left\{-2^{n-2} \sigma\right\}$ is a convergent series, the integral in (12) is finite, and Theorem 5 is proved.

\section{An example to illustrate Corollary 1}

We shall prove the following result.

Theorem 6. Suppose that $0<\alpha<1$, that $K$ is a positive integer satisfying $K>5^{2 / \alpha}$, and that

$$
f(z)=\sum_{n=0}^{\infty} K^{n(\alpha-1)} z^{K^{n}}, \quad z \in D .
$$

Then $f \in H_{a}\left(\Gamma_{K}, p\right)$ if and only if $p>1 /(1-\alpha)$. Here, $\Gamma_{K}$ is the group generated by the rotation $z \rightarrow z \exp (2 \pi i / K)$, and $H_{a}\left(\Gamma_{K}, p\right)$ is the class from Corollary 1. In particular, the classes $H_{a}\left(\Gamma_{K}, p\right)$ for different $p, 1<p<\infty$, are all distinct.

Proof. We shall denote by $C_{1}, C_{2}, \ldots$ positive constants which depend on $K$ and $\alpha$ only. For $z \in D$, we have

$$
|f(z)| \leq \sum_{n=0}^{\infty} K^{n(\alpha-1)}=\left(1-K^{\alpha-1}\right)^{-1}=C_{1} .
$$

Next we define

$$
C_{2}=\frac{\alpha \log K+\log 5}{K-1} \quad \text { and } \quad C_{3}=\frac{K(\alpha \log K-\log 5)}{K-1} .
$$

By hypothesis, $K>5^{2 / \alpha}>3$. Thus

$$
\alpha \log K>2 \log 5>\frac{(K+1) \log 5}{K-1},
$$

so that

$$
K(\alpha \log K-\log 5)>\alpha \log K+\log 5,
$$

that is, $C_{2}<C_{3}$.

For each positive integer $N$, we now define

$$
r_{N}=\exp \left(-C_{3} K^{-N}\right) \quad \text { and } \quad r_{N}^{\prime}=\exp \left(-C_{2} K^{-N}\right),
$$


and suppose that $|z|=r$, where

$$
r_{N} \leq r \leq r_{N}^{\prime}
$$

We note that

$$
z f^{\prime}(z)=\sum_{n=0}^{\infty} K^{n \alpha} z^{K^{n}}=\sum_{n=0}^{\infty} u_{N}
$$

say. Here, $\left|u_{n+1} / u_{n}\right|=K^{\alpha} r^{K^{n}(K-1)}$.

Suppose first that $n<N$. Then

$$
\begin{aligned}
\left|u_{n+1} / u_{n}\right| & =\exp \left(K^{n}(K-1) \log r+\alpha \log K\right) \\
& \geq \exp \left(-K^{N-1}(K-1) C_{3} K^{-N}+\alpha \log K\right)=5 .
\end{aligned}
$$

Thus

$$
\sum_{n=0}^{N-1}\left|u_{n}\right| \leq\left|u_{N}\right| \sum_{\nu=1}^{\infty} 5^{-\nu}=\left|u_{N}\right| / 4 .
$$

Suppose next that $n \geq N$. Then

$$
\begin{aligned}
\left|u_{n+1} / u_{n}\right| & \leq \exp \left(K^{N}(K-1) \log r+\alpha \log K\right) \\
& \leq \exp \left(-K^{N}(K-1) C_{2} K^{-N}+\alpha \log K\right)=1 / 5
\end{aligned}
$$

Thus

$$
\sum_{n=N+1}^{\infty}\left|u_{n}\right| \leq\left|u_{N}\right| \sum_{\nu=1}^{\infty} 5^{-\nu}=\left|u_{N}\right| / 4 .
$$

We deduce that in the range (19) we have

$$
\left|u_{N}\right| / 2 \leq\left|z f^{\prime}(z)\right| \leq 3\left|u_{N}\right| / 2 .
$$

In particular, when $|z|=r_{N}$ we have

$$
\left|z f^{\prime}(z)\right|<3 K^{\alpha N} / 2
$$

Thus, for $r_{N} \leq|z| \leq r_{N+1}$, we have

$$
\left|z f^{\prime}(z)\right|<3 K^{\alpha(N+1)} / 2 \leq C_{4}\left(1-r_{N}\right)^{-\alpha} \leq C_{4}(1-|z|)^{-\alpha}
$$

where $C_{4}=3\left(K C_{3}\right)^{\alpha} / 2$. Hence, since $r_{1} \geq 1 / 2$, we have for $r_{1} \leq|z|<1$,

$$
f^{\#}(z) \leq\left|f^{\prime}(z)\right| \leq 2 C_{4}(1-|z|)^{-\alpha}<2^{\alpha+1} C_{4}\left(1-|z|^{2}\right)^{-\alpha} .
$$


Suppose now that $p>1 /(1-\alpha)$. Then

$$
\begin{aligned}
& \iint_{D}\left(1-|z|^{2}\right)^{p-2}\left(f^{\#}(z)\right)^{p} d x d y \leq \\
& \iint_{|z|<r_{1}}\left(1-|z|^{2}\right)^{p-2}\left(f^{\#}(z)\right)^{p} d x d y+2 \pi\left(2^{\alpha+1} C_{4}\right)^{p} \int_{r_{1}}^{1}\left(1-r^{2}\right)^{p-2-\alpha p} r d r<\infty,
\end{aligned}
$$

so that $f \in H_{a}\left(\Gamma_{K}, p\right)$.

Next, suppose that $p \leq 1 /(1-\alpha)$ and that $|z|=r$, where $r$ lies in the range (19). Then it follows from (20) that

$$
\left|f^{\prime}(z)\right| \geq(1 / 2) K^{\alpha N} \exp \left(-C_{3}\right)
$$

Also, using (18), we deduce that

$$
f^{\#}(z)=\left|f^{\prime}(z)\right| /\left(1+|f(z)|^{2}\right) \geq C_{5} K^{\alpha N} .
$$

Thus,

$$
\iint_{r_{N}<|z|<r_{N}^{\prime}}\left(1-|z|^{2}\right)^{p-2}\left(f^{\#}(z)\right)^{p} d x d y \geq C_{6} K^{p \alpha N-(p-2) N-N} \geq C_{6}
$$

Thus

$$
\iint_{D}\left(1-|z|^{2}\right)^{p-2}\left(f^{\#}(z)\right)^{p} d x d y=\infty
$$

and the "if and only if" part of Theorem 6 is proved.

Finally, if $1<p<q<\infty$, we define $\alpha=(p-1) / p$. Then it follows that $f \in H_{a}\left(\Gamma_{K}, q\right)-H_{a}\left(\Gamma_{K}, p\right)$, so the classes $H_{a}\left(\Gamma_{K}, p\right)$ and $H_{a}\left(\Gamma_{K}, q\right)$ are distinct. This completes the proof of Theorem 6 .

\section{References}

[1] AulaskaRi, R.: Rotation-automorphic functions near the boundary. - Math. Scand. 53, 1983, 207-215.

[2] AUlaskaRI, R.: On rotation-automorphic functions with discrete rotation groups. - Ann. Acad. Sci. Fenn. Ser A I Math. 8, 1983, 371-374.

[3] Aulaskari, R.: On rotation-automorphic functions with respect to a finitely generated Fuchsian group. - J. London Math. Soc. (2) 30, 1984, 87-94.

[4] Aulaskari, R., and P. Lappan: On additive automorphic and rotation automorphic functions. - Ark. Mat. 22, 1984, 83-89.

[5] BEARDon, A.F.: The geometry of discrete groups. - In Discrete groups and automorphic functions, (Proceedings of an instructional conference organized by the London Mathematical Society and the University of Cambridge, 1975), Academic Press, 1977, $47-72$. 
[6] Drasin, D., and C.J. Earle: On the boundedness of automorphic forms. - Proc. Amer. Math. Soc. 19, 1968, 1039-1042.

[7] Dufresnoy, J.: Sur l'aire sphérique décrite par les valeurs d'une fonction méromorphe. Bull. Sci. Math. 65, 1941, 214-219.

[8] Hayman, W.K.: Regular Tsuji functions with infinitely many Julia points. - Nagoya Math. J. 29, 1967, 185-196.

[9] HaYman, W.K.: The boundary behaviour of Tsuji functions. - Michigan Math. J. 15, 1968, 1-25.

[10] KNopP, M.I.: Bounded and integrable automorphic forms. - Indiana Math. J. 22, 1973, $769-778$.

[11] LeHner, J.: On the $A_{q}(\Gamma) \subset B_{q}(\Gamma)$ conjecture. - Lecture Notes in Mathematics 320 . Springer-Verlag, 1973, 189-194.

[12] Lehner, J.: On the $A_{q}(\Gamma) \subset B_{q}(\Gamma)$ conjecture for infinitely generated groups. - Ann. of Math. Stud. 79, 1974, 283-288.

[13] Lehner, J.: On the boundedness of integrable automorphic forms. - Illinois J. Math. 18, $1974,575-585$.

[14] Lehto, O., and K.I. VirTANEN: Boundary behaviour and normal meromorphic functions. - Acta Math. 97, 1957, 47-65.

[15] Metzger, T., and K.V. RaO: On integrable and bounded automorphic forms. - Proc. Amer. Math. Soc. 28, 1971, 562-566.

[16] Metzger, T., and K.V. RaO: On integrable and bounded automorphic forms, II. - Proc. Amer. Math. Soc. 32, 1972, 201-204.

[17] Niebur, D., and M. Sheingorn: Characterization of Fuchsian groups whose integrable forms are bounded. - Ann. of Math. (2) 106, 1977, 239-258.

[18] Pommerenke, Ch.: On inclusion relations for spaces of automorphic forms. - In Advances in Complex function theory (Maryland 1973/74), Lecture Notes in Mathematics 505. Springer-Verlag, 1976, 92-100.

[19] Pommerenke, Сh.: On normal and automorphic functions. - Michigan Math. J. 21, 1974, 193-202.

\section{R. Aulaskari}

University of Joensuu

Department of Mathematics

SF-80101 Joensuu 10

Finland

Received 21 December 1988

Revised 24 October 1990
W.K. Hayman

University of York

Department of Mathematics

Heslington, York YO1 5DD

United Kingdom
P. Lappan

Michigan State University

Department of Mathematics

East Lansing, MI 48824

U.S.A. 